\title{
Stability results for 2D Navier-Stokes equations with unbounded delay
}

\author{
Linfang Liu ${ }^{\mathrm{a}, \mathrm{b}}$, Tomás Caraballo ${ }^{\mathrm{b}}$, Pedro Marín-Rubio ${ }^{\mathrm{b}}$ \\ ${ }^{a}$ School of Mathematics and Statistics, Xi'an Jiaotong University \\ Xi'an 710049, P.R. China \\ ${ }^{b}$ Dpto. Ecuaciones Diferenciales y Análisis Numérico, Universidad de Sevilla \\ C/ Tarfia s/n, 41012 Sevilla, Spain
}

\begin{abstract}
Some results related to 2D Navier-Stokes equations when the external force contains hereditary characteristics involving unbounded delays are analyzed. First, the existence and uniqueness of solutions is proved by Galerkin approximations and the energy method. The existence of stationary solution is then established by means of the Lax-Milgram theorem and the Schauder fixed point theorem. The local stability analysis of stationary solutions is studied by several different methods: the classical Lyapunov function method, the Razumikhin-Lyapunov technique and by constructing appropriate Lyapunov functionals. Finally, we also verify the polynomial stability of the stationary solution in a particular case of unbounded variable delay. Exponential stability in this infinite delay setting remains as an open problem.
\end{abstract}

Keywords: 2D Navier-Stokes equations, unbounded variable delay, stationary solution, stability, polynomial stability.

2010 MSC: 35Q30, 35A01, 35B35, 35B40.

\section{Introduction}

In this paper we investigate the following Navier-Stokes problem with unbounded delay

$$
(P)\left\{\begin{array}{l}
\frac{\partial u}{\partial t}-\nu \Delta u+(u \cdot \nabla) u+\nabla p=f(t)+g\left(t, u_{t}\right) \text { in }(0, T) \times \Omega, \\
\operatorname{div} u=0 \text { in }(0, T) \times \Omega, \\
u=0 \text { in }(0, T) \times \partial \Omega \\
u(\theta, x)=\phi(\theta, x), \theta \in(-\infty, 0], x \in \Omega,
\end{array}\right.
$$

where $\Omega \subset \mathbb{R}^{2}$ is an open and bounded set with boundary $\partial \Omega, \nu>0$ is the kinematic viscosity, $u$ is the velocity field of the fluid, $p$ denotes the pressure, $\phi$ is the initial datum, $f$ is a non-delayed external force, and $g$ is the external force containing some hereditary characteristic.

Owing to the fact that Navier-Stokes equations provide a suitable model to describe the motion of several important fluids, such as water, oil, air, etc, the long-time behavior of Navier-Stokes models (and its variants) has been regarded as an interesting and important problem in the theory of fluid dynamics, and has been receiving much attention for many years (see [16, 3, 4, 17, 38, 2] and references therein). But most results are related to non-delay situations.

Email addresses: liulinfang1988@163.com (Linfang Liu), caraball@us.es (Tomás Caraballo), pmr@us.es (Pedro Marín-Rubio) 
On the other hand, due to their importance in fluid dynamics and in turbulence theory, the Navier-Stokes equations with delay have also been extensively studied over the last years. The analysis of the Navier-Stokes equations with hereditary terms was initiated by Caraballo and Real in [14], and developed in $[11,12,13,7,15,8,9,10,5,6]$, where several issues have been investigated: the existence and uniqueness of solution, stationary solution, the existence of attractors (global, pullback and random ones) and the local exponential stability of state-steady solution of Navier-Stokes models with several types of delay (constant, bounded variable delay as well as bounded distributed delay). In the papers $[22,33,29,34,30,31,35,19,32,20,18,21]$ the authors have discussed the asymptotic behavior and regularity of solutions of 2D Navier-Stokes equations (and 3D-variations of Navier-Stokes models) with delay (finite and infinite). Wei and Zhang [39] have obtained the exponential stability and almost sure exponential stability of the weak solution for stochastic 2D Navier-Stokes equations with bounded variable delays by using the approach proposed in $[14,6]$.

It is worth emphasizing that all the mentioned works deal with finite delay (constant delay, bounded variable delay or bounded distributed delay) in the phase spaces $C([-h, 0] ; H)$ and $L^{2}(-h, 0 ; H)$ or infinite distributed delay in

$$
C_{\gamma}(H)=\left\{\varphi \in C((-\infty, 0] ; H): \lim _{\theta \rightarrow-\infty} e^{\gamma \theta} \varphi(\theta) \text { exists in } H\right\}(\gamma>0)
$$

In fact, a complete application of the theory of attractors is carried out in [35] for a 2D NavierStokes model with infinite delay in $C_{\gamma}(H)$ under some assumptions relating the force $f$ and the delay operator $g$ among others. The positive character of $\gamma$ plays a key role in those arguments. Without the help of this extra exponential weight related to the $\gamma$ parameter, we still aim to study long-time behavior of solutions to problem $(P)$, but the techniques from [35] do not seem to fit. Therefore we wonder what can be obtained if the space $C_{\gamma}(H)$ is replaced. To our best knowledge, there is no work about Navier-Stokes models with unbounded variable delay in the phase space

$$
B C L_{-\infty}(H)=\left\{\varphi \in C((-\infty, 0] ; H): \lim _{\theta \rightarrow-\infty} \varphi(\theta) \text { exists in } H\right\}
$$

which seems the natural relaxation of the precedent $C_{\gamma}(H)$ spaces. [The notation $B C L_{-\infty}$ means continuous and uniformly bounded functions with limit at $-\infty$. Actually, the term bounded is redundant when the other two conditions are imposed, but we keep this notation since in delay differential problems the space $B C$ often appears. The additional requirement of existing the limit at $-\infty$ will be clear in the existence result (cf. Theorem 3.1, step 3 of the proof).]

Inspired by $[12,35]$, in this paper we are interested in the asymptotic behavior of solutions to $2 \mathrm{D}$ Navier-Stokes equations with unbounded variable delay in the phase space $B C L_{-\infty}(H)$. More precisely, we discuss the existence, uniqueness of weak solution, and stationary solution as well as the stability of stationary solution by several different approaches. The existence and uniqueness of solution is proved by the classic Galerkin approximation and energy method, while the existence of stationary solution is established by the Schauder fixed point theorem and the Lax-Milgram theorem. Then, three methods are considered to discuss the stability of the stationary solutions. First, we study the local stability of stationary solution by using Lyapunov functions, in which the differentiability of the delay term is required, and this may seem a strong condition in some situations. Fortunately, we can use the Lyapunov-Razumikhin method to weaken the differentiability condition on the delay term, and only the continuity of the operators of the model and continuity on the delay term are necessary, which allows to include more general types of delay. By this method, but dealing with strong solution, a better result can be obtained. Besides, by constructing Lyapunov functionals, we also show the stability of stationary solution, 
and improve the result obtained by the direct approach. As a by-product we also deduce sufficient conditions for asymptotic stability. Finally, we verify, by exhibiting a special case of unbounded variable delay in an ODE, that it may not be possible, in general, to ensure exponential stability of the stationary solutions when dealing with unbounded variable delays. In fact, we are able to ensure the polynomial stability of stationary solutions in the particular case of proportional delays. Actually, for unbounded delayed ordinary differential equations the polynomial decay is a sharp result, but we use it as an upper bound here. It is still an open problem to establish some sufficient conditions ensuring asymptotic convergence to the stationary solutions with an exponential rate in general PDE problems with unbounded variable delays.

The structure and content of this paper are as follows. In Section 2, we recall some abstract spaces that will be used in this work and present some examples of forcing term with infinite delay. Section 3 is devoted to proving existence and uniqueness of solutions. In Section 4, existence and uniqueness of stationary solutions is established and several methods are used to analyze the stability of stationary solutions.

\section{Preliminaries}

To start, we consider the following usual abstract spaces,

$$
\mathcal{V}=\left\{u \in\left(C_{0}^{\infty}(\Omega)\right)^{2}: \operatorname{div} u \equiv 0\right\},
$$

$H=$ the closure of $\mathcal{V}$ in $\left(L^{2}(\Omega)\right)^{2}$ with norm $|\cdot|$, and inner product $(\cdot, \cdot)$, where for $u, v \in\left(L^{2}(\Omega)\right)^{2}$,

$$
(u, v)=\sum_{j=1}^{2} \int_{\Omega} u_{j}(x) v_{j}(x) d x,
$$

$V=$ the closure of $\mathcal{V}$ in $\left(H_{0}^{1}(\Omega)\right)^{2}$ with norm $\|\cdot\|$, and inner product $((\cdot, \cdot))$, where for $u, v \in$ $\left(H_{0}^{1}(\Omega)\right)^{2}$,

$$
((u, v))=\sum_{i, j=1}^{2} \int_{\Omega} \frac{\partial u_{j}}{\partial x_{i}} \frac{\partial v_{j}}{\partial x_{i}} d x .
$$

Identifying $H$ with its dual by the Riesz theorem, it follows that $V \subset H \equiv H^{\prime} \subset V^{\prime}$, where the injections are dense and compact. We use $\|\cdot\|_{*}$ for the norm in $V^{\prime}$ and $\langle\cdot, \cdot\rangle$ for the duality pairing between $V$ and $V^{\prime}$. Now we define $A: V \rightarrow V^{\prime}$ by $\langle A u, v\rangle=((u, v))$, and the trilinear form $b$ on $V \times V \times V$ by

$$
b(u, v, w)=\sum_{i, j=1}^{2} \int_{\Omega} u_{i} \frac{\partial v_{j}}{\partial x_{i}} w_{j} d x \forall u, v, w \in V .
$$

Let us denote $B: V \times V \rightarrow V^{\prime}$ the operator given by $\langle B(u, v), w\rangle=b(u, v, w)$, for all $u, v, w \in V$, and $B(u)=B(u, u)$.

We recall that $b(u, v, w)=-b(u, w, v)$ for all $u, v, w \in V$, and, consequently, $b(u, v, v)=0$ for all $u, v, w \in V$. Note that after Ladyzhenskaya inequality [28], the trilinear form $b$ satisfies the following one, which will be used later in proofs,

$$
\begin{aligned}
|b(u, v, u)| & \leq\|u\|_{\left(L^{4}(\Omega)\right)^{2}}^{2}\|v\| \\
& \leq 2^{-1 / 2}|u|\|u\|\|v\| \forall u, v \in V .
\end{aligned}
$$


There are several phase spaces which allow us to deal with infinite delays (e.g., cf. [36, 25, 24]). As we commented in the introduction, we aim to establish well-posedness and stability results for 2D Navier-Stokes equations with infinite delay operators in the phase space

$$
B C L_{-\infty}(H)=\left\{\varphi \in C((-\infty, 0] ; H): \lim _{\theta \rightarrow-\infty} \varphi(\theta) \text { exists in } H\right\},
$$

which is a Banach space equipped with the norm

$$
\|\varphi\|_{B C L_{-\infty}(H)}=\sup _{\theta \in(-\infty, 0]}|\varphi(\theta)| .
$$

Let us introduce some notation and assumptions on the delay operator. We will denote $\mathbb{R}_{+}=$ $[0, \infty)$. Let $X$ be a Banach space and consider a fixed $T>0$. Given $u:(-\infty, T) \rightarrow X$, for each $t \in(0, T)$, we denote by $u_{t}$ the function defined on $(-\infty, 0]$ by

$$
u_{t}(\theta)=u(t+\theta), \quad \theta \in(-\infty, 0] .
$$

We now enumerate the assumptions on the delay term $g$. Assume that $g:[0, T] \times B C L_{-\infty}(H) \rightarrow$ $\left(L^{2}(\Omega)\right)^{2}$, then

$(g 1)$ For any $\xi \in B C L_{-\infty}(H)$, the mapping $[0, T] \ni t \mapsto g(t, \xi) \in\left(L^{2}(\Omega)\right)^{2}$ is measurable.

$(g 2) g(\cdot, 0)=0$.

(g3) There exists a constant $L_{g}>0$ such that, for any $t \in[0, T]$ and all $\xi, \eta \in B C L_{-\infty}(H)$,

$$
|g(t, \xi)-g(t, \eta)| \leq L_{g}\|\xi-\eta\|_{B C L_{-\infty}(H)}
$$

Remark 2.1. (i) As it is pointed out in [35], condition ( $g 2)$ is not really a restriction, otherwise, if $|g(\cdot, 0)| \in L^{2}(0, T)$, we could redefine $\hat{f}(t)=f(t)+g(t, 0)$ and $\hat{g}(t, \cdot)=g(t, \cdot)-g(t, 0)$. In this way, the problem is exactly the same, but $\hat{f}$ and $\hat{g}$ satisfy the required assumptions.

(ii) Conditions (g2) and (g3) imply that, for any $\xi \in B C L_{-\infty}(H)$,

$$
|g(t, \xi)| \leq L_{g}\|\xi\|_{B C L_{-\infty}(H)} \forall t \in[0, T]
$$

and therefore $|g(\cdot, \xi)| \in L^{\infty}(0, T)$.

Now we provide some examples of (unbounded) delay forcing terms which can be set within our general set-up (see $[22,19,20,18,21])$.

Example 2.2 (Forcing term with unbounded variable delay). Let $G:[0, T] \times \mathbb{R}^{2} \rightarrow \mathbb{R}^{2}$ be a measurable function satisfying $G(t, 0)=0$ for all $t \in[0, T]$, and assume that there exists $M>0$ such that

$$
|G(t, u)-G(t, v)|_{\mathbb{R}^{2}} \leq M|u-v|_{\mathbb{R}^{2}} \forall u, v \in \mathbb{R}^{2}
$$

Consider a function $\rho:[0, T] \rightarrow \mathbb{R}_{+}$, which plays the role of the delay. Assume that $\rho(\cdot)$ is measurable and define $g(t, \xi)(x)=G(t, \xi(-\rho(t))(x))$ for each $\xi \in B C L_{-\infty}(H), x \in \Omega$ and $t \in$ $[0, T]$. 
Obviously, $g$ satisfies $(g 1)-(g 2)$. Now we check that $g$ satisfies assumption (g3), for any $\xi, \eta \in B C L_{-\infty}(H)$,

$$
\begin{aligned}
|g(t, \xi)-g(t, \eta)|^{2} & =\int_{\Omega}|G(t, \xi(-\rho(t)))-G(t, \eta(-\rho(t)))|_{\mathbb{R}^{2}}^{2} d x \\
& \leq M^{2} \int_{\Omega}|\xi(-\rho(t))-\eta(-\rho(t))|_{\mathbb{R}^{2}}^{2} d x \\
& \leq M^{2} \sup _{\theta \leq 0} \int_{\Omega}|\xi(\theta)-\eta(\theta)|_{\mathbb{R}^{2}}^{2} d x \\
& =M^{2}\|\xi-\eta\|_{B C L_{-\infty}(H)}^{2} .
\end{aligned}
$$

Example 2.3. The above example is using the mapping $G$ via the Nemytskii operator to deal with an operator from $[0, T] \times H$ into $\left(L^{2}(\Omega)\right)^{2}$. Thus, it is a particular case of the following. Take a Lipschitz mapping (uniformly w.r.t. $[0, T]) G:[0, T] \times H \rightarrow\left(L^{2}(\Omega)\right)^{2}$ and consider $g(t, \xi):=G\left(t, \xi(-\rho(t))\right.$ for any measurable function $\rho:[0, T] \rightarrow \mathbb{R}_{+}$. This operator $g$ also fulfils $(g 1)-(g 3)$.

Example 2.4 (Forcing term with distributed delay). Let $G:[0, T] \times(-\infty, 0] \times \mathbb{R}^{2} \rightarrow \mathbb{R}^{2}$ be a measurable function satisfying $G(t, s, 0)=0$ for all $(t, s) \in[0, T] \times(-\infty, 0]$, and suppose that there exists a function $\alpha \in L^{1}(-\infty, 0)$ such that

$$
|G(t, s, u)-G(t, s, v)|_{\mathbb{R}^{2}} \leq \alpha(s)|u-v|_{\mathbb{R}^{2}} \forall u, v \in \mathbb{R}^{2}, \forall t \in[0, T] \text {, a.e. } s \in(-\infty, 0) .
$$

Define $g(t, \xi)(x)=\int_{-\infty}^{0} G(t, s, \xi(s)(x)) d s$ for each $\xi \in B C L_{-\infty}(H), t \in[0, T]$, and $x \in \Omega$. Then the delayed term $g$ in our problem becomes

$$
g(t, \xi)=\int_{-\infty}^{0} G(t, s, \xi(s)) d s .
$$

It is easy to check that $g$ satisfies $(g 1)-(g 2)$. On the other hand, if $\xi, \eta \in B C L_{-\infty}(H)$, for each $t \in[0, T]$, we deduce

$$
\begin{aligned}
|g(t, \xi)-g(t, \eta)|^{2} & \leq \int_{\Omega}\left(\int_{-\infty}^{0}|G(t, s, \xi(s)(x))-G(t, s, \eta(s)(x))|_{\mathbb{R}^{2}} d s\right)^{2} d x \\
& \leq \int_{\Omega}\left(\int_{-\infty}^{0} \alpha(s)|\xi(s)(x)-\eta(s)(x)|_{\mathbb{R}^{2}} d s\right)^{2} d x \\
& \leq \int_{\Omega}\left(\int_{-\infty}^{0} \alpha(s) d s\right)\left(\int_{-\infty}^{0} \alpha(s)|\xi(s)(x)-\eta(s)(x)|_{\mathbb{R}^{2}}^{2} d s\right) d x \\
& \leq\|\alpha\|_{L^{1}(-\infty, 0)} \int_{-\infty}^{0} \alpha(s) \int_{\Omega}|\xi(s)(x)-\eta(s)(x)|^{2} d x d s \\
& \leq\|\alpha\|_{L^{1}(-\infty, 0)} \int_{-\infty}^{0} \alpha(s)\left(\sup _{s \leq 0} \int_{\Omega}|\xi(s)(x)-\eta(s)(x)|^{2} d x\right) d s \\
& \leq\|\alpha\|_{L^{1}(-\infty, 0)}^{2}\|\xi-\eta\|_{B C L_{-\infty}(H)}^{2} .
\end{aligned}
$$

After introducing the above operators an equivalent abstract formulation to problem $(P)$ is

$$
\begin{aligned}
& \frac{d u}{d t}+\nu A u+B(u)=P f+P g\left(t, u_{t}\right) \forall t \geq 0, \\
& u_{0}=\phi
\end{aligned}
$$


where $P$ is the Leray projector from $\left(L^{2}(\Omega)\right)^{2}$ onto $H$.

Next we establish the definition of weak solution to problem (2.2)-(2.3).

Definition 2.5. Given an initial datum $\phi \in B C L_{-\infty}(H)$, a weak solution $u$ to (2.2)-(2.3) in the interval $(-\infty, T]$ is a function $u \in C((-\infty, T] ; H) \cap L^{2}(0, T ; V)$ with $u_{0}=\phi$ such that, for all $v \in V$,

$$
\frac{d}{d t}(u(t), v)+\nu((u(t), v))+b(u(t), u(t), v)=\langle f(t), v\rangle+\left(g\left(t, u_{t}\right), v\right),
$$

where the equation must be understood in the sense of $\mathcal{D}^{\prime}(0, T)$.

\section{Existence, uniqueness and regularity of solutions}

In this section we establish the existence of weak solution to (2.2)-(2.3) by a compactness method using the classic Faedo-Galerkin scheme. Denote

$$
\lambda_{1}=\inf _{v \in V \backslash\{0\}} \frac{\|v\|^{2}}{|v|^{2}}>0 .
$$

For the existence of weak solution we have the following result.

Theorem 3.1. Consider $f \in L^{2}\left(0, T ; V^{\prime}\right), g:[0, T] \times B C L_{-\infty}(H) \rightarrow\left(L^{2}(\Omega)\right)^{2}$ satisfying $(g 1)-(g 3)$ and $\phi \in B C L_{-\infty}(H)$ given. Then there exists a unique weak solution to (2.2)(2.3). Furthermore, if $f \in L^{2}\left(0, T ;\left(L^{2}(\Omega)\right)^{2}\right)$ and $\phi \in B C L_{-\infty}(H)$ with $\phi(0) \in V$, then the weak solution is a strong solution, i.e., $u \in L^{2}(0, T ; D(A)) \cap C([0, T] ; V)$.

Proof. We split it into several steps.

Step 1. The Galerkin approximation. By the definition of $A$ and the classical spectral theory of elliptic operators, it follows that $A$ possesses a sequence of eigenvalues $\left\{\lambda_{j}\right\}_{j \geq 1}$ and a corresponding family of eigenfunctions $\left\{w_{j}\right\}_{j \geq 1} \subset V$, which form a Hilbert basis of $H$, dense on $V$. We consider the subspace $V_{m}=\operatorname{span}\left\{w_{1}, w_{2}, \cdots, w_{m}\right\}$, and the projector $P_{m}: H \rightarrow V_{m}$ given by $P_{m} u=\sum_{j=1}^{m}\left(u, w_{j}\right) w_{j}$, and define $u^{(m)}(t)=\sum_{j=1}^{m} \gamma_{m, j}(t) w_{j}$, where the superscript $m$ will be used instead of $(m)$, for short, since no confusion is possible with powers of $u$, and where the coefficients $\gamma_{m, j}(t)$ are required to satisfy the Cauchy problem

$$
\begin{aligned}
& \frac{d}{d t}\left(u^{m}(t), w_{j}\right)+\nu\left(\left(u^{m}(t), w_{j}\right)\right)+b\left(u^{m}(t), u^{m}(t), w_{j}\right)=\left\langle f(t), w_{j}\right\rangle+\left(g\left(t, u_{t}^{m}\right), w_{j}\right), \quad 1 \leq j \leq m, \\
& u^{m}(\theta)=P_{m} \phi(\theta), \theta \in(-\infty, 0] .
\end{aligned}
$$

The above system of ordinary functional differential equations with infinite delay fulfills the conditions for the existence and uniqueness of local solutions (e.g., cf. [23, 37, 25]). Hence, we conclude that (3.1) has a unique local solution defined in $\left[0, t_{m}\right)$ with $0 \leq t_{m} \leq T$. Next, we will obtain a priori estimates and ensure that the solutions $u^{m}$ do exist in the whole interval $[0, T]$.

Step 2. A priori estimates. Multiplying each equation of $(3.1)$ by $\gamma_{m, j}(t), j=1, \ldots, m$, summing up, and using Cauchy-Schwartz and Young's inequalities, we obtain

$$
\begin{aligned}
\frac{1}{2} \frac{d}{d t}\left|u^{m}(t)\right|^{2}+\nu\left\|u^{m}(t)\right\|^{2} & \leq\|f(t)\|_{*}\left\|u^{m}(t)\right\|+L_{g}\left\|u_{t}^{m}\right\|_{B C L_{-\infty}(H)}\left|u^{m}(t)\right| \\
& \leq \frac{\nu}{2}\left\|u^{m}(t)\right\|^{2}+\frac{\|f(t)\|_{*}^{2}}{2 \nu}+L_{g}\left\|u_{t}^{m}\right\|_{B C L_{-\infty}(H)}^{2} .
\end{aligned}
$$


Hence,

$$
\left|u^{m}(t)\right|^{2}+\nu \int_{0}^{t}\left\|u^{m}(s)\right\|^{2} d s \leq\left|u^{m}(0)\right|^{2}+\frac{1}{\nu} \int_{0}^{t}\|f(s)\|_{*}^{2} d s+2 L_{g} \int_{0}^{t}\left\|u_{s}^{m}\right\|_{B C L_{-\infty}(H)}^{2} d s .
$$

In particular

$$
\left\|u_{t}^{m}\right\|_{B C L_{-\infty}(H)}^{2} \leq\|\phi\|_{B C L_{-\infty}(H)}^{2}+\frac{1}{\nu} \int_{0}^{t}\|f(s)\|_{*}^{2} d s+2 L_{g} \int_{0}^{t}\left\|u_{s}^{m}\right\|_{B C L_{-\infty}(H)}^{2} d s .
$$

Applying now the Gronwall lemma,

$$
\left\|u_{t}^{m}\right\|_{B C L_{-\infty}(H)}^{2} \leq\left(\|\phi\|_{B C L_{-\infty}(H)}^{2}+\frac{1}{\nu} \int_{0}^{t}\|f(s)\|_{*}^{2} d s\right) e^{2 L_{g} t}
$$

whence there exists a constant $C>0$, depending on some constants of the problem (namely, $\nu, L_{g}$ and $f$ ), and on $T$ and $R>0$, such that

$$
\left\|u_{t}^{m}\right\|_{B C L_{-\infty}(H)}^{2} \leq C(T, R) \forall t \in[0, T], \quad\|\phi\|_{B C L_{-\infty}(H)} \leq R, \quad \forall m \geq 1,
$$

which also implies that $\left\{u^{m}\right\}$ is bounded in $L^{\infty}(0, T ; H)$.

Now it follows from (3.2) and the above uniform estimates that

$$
\nu \int_{0}^{t}\left\|u^{m}(s)\right\|^{2} d s \leq\left|u^{m}(0)\right|^{2}+\int_{0}^{t}\left(\frac{1}{\nu}\|f(s)\|_{*}^{2}+2 L_{g} C(T, R)\right) d s .
$$

We can conclude the existence of another constant (relabelled the same) $C(T, R)$ such that

$$
\left\|u^{m}\right\|_{L^{2}(0, T ; V)}^{2} \leq C(T, R) \forall m \geq 1
$$

From (2.1) and (3.1),

$$
\left\|\left(u^{m}\right)^{\prime}\right\|_{*} \leq \nu\left\|u^{m}\right\|+2^{1 / 2}\left|u^{m}\right|\left\|u^{m}\right\|+\|f\|_{*}+\lambda_{1}^{-1 / 2}\left|g\left(t, u_{t}^{m}\right)\right|,
$$

which, together with Remark 2.1(ii) and the above estimates imply that $\left\{\left(u^{m}\right)^{\prime}\right\}$ is bounded in $L^{2}\left(0, T ; V^{\prime}\right)$.

Step 3. Approximation of initial datum in $B C L_{-\infty}(H)$. Let us prove that

$$
P_{m} \phi \rightarrow \phi \text { in } B C L_{-\infty}(H) \text {. }
$$

Indeed, if not, there exist $\epsilon>0$ and a subsequence $\left\{\theta_{m}\right\} \subset(-\infty, 0]$, such that

$$
\left|P_{m} \phi\left(\theta_{m}\right)-\phi\left(\theta_{m}\right)\right|>\epsilon \forall m
$$

We claim that $\theta_{m} \rightarrow-\infty$. Otherwise, if $\theta_{m} \rightarrow \theta$, then $P_{m} \phi\left(\theta_{m}\right) \rightarrow \phi(\theta)$, since $\left|P_{m} \phi\left(\theta_{m}\right)-\phi(\theta)\right| \leq$ $\left|P_{m} \phi\left(\theta_{m}\right)-P_{m} \phi(\theta)\right|+\left|P_{m} \phi(\theta)-\phi(\theta)\right| \rightarrow 0$ as $m \rightarrow \infty$. Now, since $\theta_{m} \rightarrow-\infty$ as $m \rightarrow \infty$, if we denote $x=\lim _{\theta \rightarrow-\infty} \phi(\theta)$, we obtain

$$
\left|P_{m} \phi\left(\theta_{m}\right)-\phi\left(\theta_{m}\right)\right| \leq\left|P_{m} \phi\left(\theta_{m}\right)-P_{m} x\right|+\left|P_{m} x-x\right|+\left|x-\phi\left(\theta_{m}\right)\right| \rightarrow 0,
$$

which contradicts (3.4), and thus (3.3) holds true. 
Step 4. Compactness results. Following the same lines as those in [35, Theorem 5, p.2017] with slight modifications, we can prove that

$$
u^{m} \rightarrow u \text { in } C([0, T] ; H) .
$$

Then steps 3 and 4 imply that

$$
u_{t}^{m} \rightarrow u_{t} \text { in } B C L_{-\infty}(H) \forall t \leq T .
$$

Actually,

$$
\begin{aligned}
\sup _{\theta \leq 0}\left|u^{m}(t+\theta)-u(t+\theta)\right| & =\max \left\{\sup _{\theta \leq-t}\left|P_{m} \phi(\theta+t)-\phi(\theta+t)\right|, \sup _{-t \leq \theta \leq 0}\left|u^{m}(t+\theta)-u(t+\theta)\right|\right\} \\
& \leq \max \left\{\left\|P_{m} \phi-\phi\right\|_{B C L_{-\infty}(H)}, \sup _{-t \leq \theta \leq 0}\left|u^{m}(t+\theta)-u(t+\theta)\right|\right\} \rightarrow 0 .
\end{aligned}
$$

Therefore, taking into account $(g 3)$, we can prove that

$$
g\left(\cdot, u_{.}^{m}\right) \rightarrow g(\cdot, u .) \text { in } L^{2}(0, T ; H) .
$$

Thus, we can finally pass to the limit in (3.1), concluding that $u$ solves $(P)$.

Step 5. Uniqueness of solution. The uniqueness of solution can be obtained by using the Gronwall Lemma. Namely, consider two solutions $u$ and $v$ to (2.2)-(2.3), and denote by $w=u-v$. Using the properties of $b$ we have that (see $[35,6]$ for more details)

$$
|b(u(t), u(t), u(t)-v(t))-b(v(t), v(t), u(t)-v(t))|=|b(w(t), u(t), w(t))| .
$$

Using the energy equality in the equation satisfied by $w$, combined with $(g 3)$ and $(2.1)$,

$$
\begin{aligned}
|w(t)|^{2}+2 \nu \int_{0}^{t}\|w(s)\|^{2} d s & =-2 \int_{0}^{t} b(w(s), u(s), w(s)) d s+2 \int_{0}^{t}\left(g\left(s, u_{s}\right)-g\left(s, v_{s}\right), w(s)\right) d s \\
& \leq 2^{1 / 2} \int_{0}^{t}|w(s)|\|w(s)\|\|u(s)\| d s+2 L_{g} \int_{0}^{t}\left\|w_{s}\right\|_{B C L_{-\infty}(H)}|w(s)| d s .
\end{aligned}
$$

By the Young inequality and the definition of the $B C L_{-\infty}(H)$-norm,

$$
|w(t)|^{2} \leq \frac{1}{4 \nu} \int_{0}^{t}\|u(s)\|^{2}|w(s)|^{2} d s+2 L_{g} \int_{0}^{t}\left\|w_{s}\right\|_{B C L_{-\infty}(H)}^{2} d s \forall t \in[0, T] .
$$

Since $w(\theta)=0$ for $\theta \leq 0$, taking the maximum in $[0, t]$ for any $t \in[0, T]$,

$$
\left\|w_{t}\right\|_{B C L_{-\infty}(H)}^{2} \leq\left(\frac{1}{4 \nu}+2 L_{g}\right) \int_{0}^{t}\left(1+\|u(s)\|^{2}\right)\left\|w_{s}\right\|_{B C L_{-\infty}(H)}^{2} d s
$$

whence the Gronwall Lemma gives $w \equiv 0$.

Step 6. Strong solution. Once that $f \in L^{2}\left(0, T ;\left(L^{2}(\Omega)\right)^{2}\right)$ and $\phi \in B C L_{-\infty}(H)$ with $\phi(0) \in$ $V$, it is immediate to verify that for $u$ the solution to $(2.2)-(2.3), f(\cdot)+g(\cdot, u.) \in L^{2}\left(0, T ;\left(L^{2}(\Omega)\right)^{2}\right)$. Now, it is a standard matter to gain the claimed (strong) regularity for $u$, as a solution to a nondelayed Navier-Stokes problem (e.g., cf. [38]) with regular right-hand side. 


\section{Asymptotic behavior of solutions}

In this section we analyze the long time behavior of solutions in a neighborhood of a stationary solution to (2.2) in some particular settings for the delay operator. First of all, we provide several results ensuring the existence and eventual uniqueness of stationary solutions. Then we show various methods to study the stability properties: the Lyapunov function method, the Razumikhin technique as well as the construction method of appropriate Lyapunov functionals. All the cases will be related to model (2.2) with unbounded variable delays. We would also like to mention that for particular unbounded variable delays, the exponential stability of stationary solutions for delayed ODE cannot be obtained (cf. [1]). However, inspired in those results, we will be able to obtain some polynomial stability for problem $(\mathrm{P})$ in the case of proportional variable delays.

\subsection{Existence and uniqueness of stationary solution}

In order to investigate the existence and properties of stationary solutions to (2.2), we need to impose some extra assumptions. Namely, we assume that $f$ is independent of time, i.e., $f(t) \equiv f \in V^{\prime}$, and $g$, defined now for all positive times, also is autonomous somehow. Indeed, if we assume directly $g$ to be autonomous, then the delay should have a distributed or fixed form, but an infinite variable delay would not be possible. Therefore the explicit presence of $t$ in the operator should not be removed if we aim to keep the variable delay case within our set-up. Namely, we introduce a new assumption for $g$. Denote by $i$ the trivial immersion $i: H \rightarrow B C L_{-\infty}(H)$ given by $i(u)=\tilde{u}$ with $\tilde{u}(t)=u$ for all $t \leq 0$. We require now that $g$ fulfills

$(g 4) g(s, \xi)=g(t, \xi)$ for any $s, t \in \mathbb{R}_{+}$and $\xi \in i(H)$.

If $(g 2)-(g 4)$ holds, we trivially have that $\tilde{g}: H \rightarrow\left(L^{2}(\Omega)\right)^{2}$ defined as $\tilde{g}(u)=g(0, i(u))$, i.e., $\tilde{g}=\left.g\right|_{\mathbb{R}_{+} \times i(H)}$, is of course autonomous, Lipschitz (with the same Lipschitz constant $L_{g}$ ) and $\tilde{g}(0)=0$.

Example 4.1. Combining Examples 2.2 and 2.3 it is obvious that given a measurable function $\rho: \mathbb{R}_{+} \rightarrow \mathbb{R}_{+}$and $G: H \rightarrow\left(L^{2}(\Omega)\right)^{2}$ Lipschitz with $G(0)=0$, then $\mathbb{R}_{+} \times B C L_{-\infty}(H) \ni(t, \xi) \mapsto$ $g(t, \xi):=G(\xi(-\rho(t))) \in\left(L^{2}(\Omega)\right)^{2}$ fulfills $(g 1)-(g 4)$.

A stationary solution to $(2.2)$ is a function $u^{*} \in V$ such that

$$
\nu A u^{*}+B\left(u^{*}\right)=P f+P \tilde{g}\left(u^{*}\right) .
$$

Notice that (4.1) is not related to any delay form, and has already been analyzed in some previous works (e.g., cf. [12, 14, 22, 35, 6]). Existence, eventual uniqueness and regularity of stationary solutions can be obtained as stated in the next result.

Theorem 4.2. Suppose that $g$ satisfies conditions $(g 2)-(g 4)$ and $\nu>\lambda_{1}^{-1} L_{g}$. Then,

(a) for all $f \in V^{\prime}$, there exists at least one solution to (4.1);

(b) if $f \in\left(L^{2}(\Omega)\right)^{2}$, the solutions to (4.1) belong to $D(A)$;

(c) if $\left(\nu-\lambda_{1}^{-1} L_{g}\right)^{2}>\left(2 \lambda_{1}\right)^{-1 / 2}\|f\|_{*}$, then the solution to (4.1) is unique.

As commented in the introduction of this section, our goal for the rest of the paper is to analyze stability conditions for stationary solutions to (2.2). To be precise, the notions we will use are the following (e.g., cf. [15]). 
Definition 4.3. A stationary solution $u^{*}$ to (2.2) is stable if for any $\varepsilon>0$ there exists $\delta>0$ such that if $\phi \in B C L_{-\infty}(H)$ satisfies $\left\|\phi-i\left(u^{*}\right)\right\|_{B C L_{-\infty}(H)}<\delta$, then the solution $u(\cdot ; \phi)$ to (2.2)-(2.3) exists for all $t \geq 0$ and satisfies $\left|u(t ; \phi)-u^{*}\right|<\varepsilon$ for any $t \geq 0$.

A stationary solution $u^{*}$ to (2.2) is said to be attractive if there exists $\widetilde{\delta}>0$ such that if $\phi \in B C L_{-\infty}(H)$ satisfies $\left\|\phi-i\left(u^{*}\right)\right\|_{B C L_{-\infty}(H)}<\widetilde{\delta}$, then the solution $u(\cdot ; \phi)$ to (2.2)-(2.3) exists for all $t \geq 0$ and satisfies $\lim _{t \rightarrow \infty}\left|u(t ; \phi)-u^{*}\right|=0$.

A stationary solution $u^{*}$ to (2.2) is said to be asymptotically stable if it is stable and attractive.

\subsection{Local stability: a direct approach}

In this section we prove the local stability of stationary solution obtained in Theorem 4.2 when $g$ is close to that in Example 4.1 (with a smoother driving term $\rho$ ) by a direct approach.

Theorem 4.4. Consider $f \in\left(L^{2}(\Omega)\right)^{2}$, the delay forcing term given by $g\left(t, u_{t}\right)=G(u(t-\rho(t)))$ with $G: H \rightarrow\left(L^{2}(\Omega)\right)^{2}$ a Lipschitz operator with Lipschitz constant $M, G(0)=0$, and $\rho \in$ $C^{1}\left(\mathbb{R}_{+}, \mathbb{R}_{+}\right)$with $\rho_{*}=\sup _{t \geq 0} \rho^{\prime}(t)<1$. There exist constants $l_{1}, l_{2}$, depending only on $\Omega$, and $C=C\left(\rho_{*}, M\right)$, such that if

$$
2 \nu>\frac{\left(2-\rho_{*}\right) \lambda_{1}^{-1} M}{1-\rho_{*}}+\frac{l_{1}}{\nu-\lambda_{1}^{-1} M}|f|+\frac{l_{2}}{\nu^{2}\left(\nu-\lambda_{1}^{-1} M\right)^{3}}|f|^{3},
$$

then, for any solution $u_{\infty} \in D(A)$ to (4.1) (whose existence is guaranteed by Theorem 4.2), and any $\phi \in B C L_{-\infty}(H)$, the solution $u$ to (2.2)-(2.3) with $f(t) \equiv f$ satisfies

$$
\left|u(t)-u_{\infty}\right|^{2} \leq C\left(\left|\phi(0)-u_{\infty}\right|^{2}+\left\|\phi-u_{\infty}\right\|_{L^{2}((-\rho(0), 0) ; H)}^{2}\right) \forall t \geq 0 .
$$

Proof. Consider $u$ the solution to (2.2)-(2.3) for $f(t) \equiv f$ and let $u_{\infty} \in D(A)$ be a solution to (4.1) (this is guaranteed by Theorem 4.2 since (4.2) implies that $\nu>\lambda_{1}^{-1} M=\lambda_{1}^{-1} L_{g}$ ). We set $w(t)=u(t)-u_{\infty}$, and observe that

$$
\frac{d}{d t} w(t)+\nu A w(t)+B(u(t))-B\left(u_{\infty}\right)=P G(u(t-\rho(t)))-P G\left(u_{\infty}\right) .
$$

By standard computations,

$$
\begin{aligned}
\frac{d}{d t}|w(t)|^{2} & =-2 \nu\|w(t)\|^{2}-2\left(B(u(t))-B\left(u_{\infty}\right), w(t)\right)+2\left(G(u(t-\rho(t)))-G\left(u_{\infty}\right), w(t)\right) \\
& \leq-2 \nu\|w(t)\|^{2}-2 b\left(w(t), u_{\infty}, w(t)\right)+2 M\left|u(t-\rho(t))-u_{\infty}\right||w(t)| \\
& \leq\left(-2 \nu+\lambda_{1}^{-1} M\right)\|w(t)\|^{2}-2 b\left(w(t), u_{\infty}, w(t)\right)+M|w(t-\rho(t))|^{2} .
\end{aligned}
$$

By (2.1), and using the Sobolev embeddings (introducing suitable constants $c_{0}, c_{0}^{\prime}, c_{1}$ ), we have

$$
\begin{aligned}
2\left|b\left(w(t), u_{\infty}, w(t)\right)\right| & \leq 2|w(t)|_{\left(L^{4}(\Omega)\right)^{2}}^{2}\left\|u_{\infty}\right\| \\
& \leq c_{0} 2^{1 / 2} \lambda_{1}^{-1 / 2}\|w(t)\|^{2}\left|A u_{\infty}\right| .
\end{aligned}
$$

Since $u_{\infty}$ solves (4.1), we deduce

$$
\begin{aligned}
\nu\left|A u_{\infty}\right| & \leq|f|+\left|G\left(u_{\infty}\right)\right|+\left|B\left(u_{\infty}\right)\right| \\
& \leq|f|+M\left|u_{\infty}\right|+c_{0}^{\prime}\left\|u_{\infty}\right\|\left|u_{\infty}\right|_{\infty} \\
& \leq|f|+M\left|u_{\infty}\right|+c_{1}\left\|u_{\infty}\right\|\left|u_{\infty}\right|^{1 / 2}\left|A u_{\infty}\right|^{1 / 2} \\
& \leq|f|+M\left|u_{\infty}\right|+c_{1} \lambda_{1}^{-1 / 4}\left\|u_{\infty}\right\|^{3 / 2}\left|A u_{\infty}\right|^{1 / 2} \\
& \leq|f|+\lambda_{1}^{-1 / 2} M\left\|u_{\infty}\right\|+\frac{c_{1}^{2} \lambda_{1}^{-1 / 2}}{2 \nu}\left\|u_{\infty}\right\|^{3}+\frac{\nu}{2}\left|A u_{\infty}\right|
\end{aligned}
$$


from which we obtain that

$$
\left|A u_{\infty}\right| \leq \frac{2}{\nu}|f|+\frac{2 \lambda_{1}^{-1 / 2} M}{\nu}\left\|u_{\infty}\right\|+\frac{c_{1}^{2} \lambda_{1}^{-1 / 2}}{\nu^{2}}\left\|u_{\infty}\right\|^{3} .
$$

On the other hand,

$$
\begin{aligned}
\nu\left\|u_{\infty}\right\|^{2} & =\left(f, u_{\infty}\right)+\left(G\left(u_{\infty}\right), u_{\infty}\right) \\
& \leq \lambda_{1}^{-1 / 2}|f|\left\|u_{\infty}\right\|+\lambda_{1}^{-1} M\left\|u_{\infty}\right\|^{2}
\end{aligned}
$$

which implies

$$
\left\|u_{\infty}\right\| \leq \frac{\lambda_{1}^{-1 / 2}|f|}{\nu-\lambda_{1}^{-1} M}
$$

Hence from the above inequalities,

$$
\left|A u_{\infty}\right| \leq \frac{2}{\nu}|f|+\frac{2 \lambda_{1}^{-1} M}{\nu\left(\nu-\lambda_{1}^{-1} M\right)}|f|+\frac{c_{1}^{2} \lambda_{1}^{-2}}{\nu^{2}\left(\nu-\lambda_{1}^{-1} M\right)^{3}}|f|^{3} .
$$

Now, thanks to the previous inequalities,

$$
\frac{d}{d t}|w(t)|^{2} \leq\left(-2 \nu+\lambda_{1}^{-1} M+\frac{l_{1}}{\nu-\lambda_{1}^{-1} M}|f|+\frac{l_{2}}{\nu^{2}\left(\nu-\lambda_{1}^{-1} M\right)^{3}}|f|^{3}\right)\|w(t)\|^{2}+M|w(t-\rho(t))|^{2},
$$

where

$$
l_{1}=c_{0} 2^{3 / 2} \lambda_{1}^{-1 / 2}, \quad l_{2}=2^{1 / 2} c_{0} c_{1}^{2} \lambda_{1}^{-5 / 2} .
$$

Taking $\eta=s-\rho(s)=\tau(s)$,

$$
M \int_{0}^{t}|w(s-\rho(s))|^{2} d s \leq \frac{M}{1-\rho_{*}} \int_{-\rho(0)}^{t}|w(\eta)|^{2} d \eta .
$$

Therefore, integrating (4.4) over $[0, t]$,

$$
\begin{aligned}
|w(t)|^{2} \leq & |w(0)|^{2}+\int_{0}^{t}\left(\frac{\lambda_{1}^{-1} M}{1-\rho_{*}}-2 \nu+\lambda_{1}^{-1} M+\frac{l_{1}}{\nu-\lambda_{1}^{-1} M}|f|+\frac{l_{2}}{\nu^{2}\left(\nu-\lambda_{1}^{-1} M\right)^{3}}|f|^{3}\right)\|w(s)\|^{2} d s \\
& +\frac{M}{1-\rho_{*}} \int_{-\rho(0)}^{0}|w(s)|^{2} d s
\end{aligned}
$$

which, together with (4.2), yield

$$
|w(t)|^{2} \leq|w(0)|^{2}+\frac{M}{1-\rho_{*}} \int_{-\rho(0)}^{0}|w(s)|^{2} d s,
$$

whence the statement follows taking $C=\max \left\{1, M /\left(1-\rho_{*}\right)\right\}$.

Remark 4.5. Observe that there exists at least one stationary solution under assumptions of Theorem 4.4, but it might not be unique since the relation on the coefficients are different from Theorem 4.2 (c), which ensures the uniqueness of stationary solution. However, if $2\left(2 \lambda_{1}\right)^{-1 / 4}\|f\|_{*}^{1 / 2} \leq$ $\frac{\lambda_{1}^{-1} M \rho_{*}}{1-\rho_{*}}+\frac{l_{1}}{\nu-\lambda_{1}^{-1} M}|f|+\frac{l_{2}}{\nu^{2}\left(\nu-\lambda_{1}^{-1} M\right)^{3}}|f|^{3}$, then Theorem 4.2 (c) implies that the stationary solution is unique. 
Remark 4.6. Notice that, even assuming a relationship among the structural constants of the problem to guarantee uniqueness (cf. Remark 4.5), if we wish to obtain exponential stability by the Lyapunov method, we can multiply (4.3) by $e^{\lambda t}$. Then by a similar process with slight modification, we would obtain

$$
\begin{aligned}
e^{\lambda t}|w(t)|^{2} \leq & |w(0)|^{2}+\int_{0}^{t}\left(\lambda \lambda_{1}^{-1}-2 \nu+\lambda_{1}^{-1} M+\frac{l_{1}}{\nu-\lambda_{1}^{-1} M}|f|+\frac{l_{2}}{\nu^{2}\left(\nu-\lambda_{1}^{-1} M\right)^{3}}|f|^{3}\right) e^{\lambda s}\|w(s)\|^{2} d s \\
& +M \int_{0}^{t} e^{\lambda s}|w(s-\rho(s))|^{2} d s .
\end{aligned}
$$

Now we estimate the delay term. Setting $\eta=s-\rho(s)=\tau(s)$,

$$
\int_{0}^{t} e^{\lambda s}|w(s-\rho(s))|^{2} d s \leq \frac{1}{1-\rho_{*}} \int_{-\rho(0)}^{t-\rho(t)} e^{\lambda \tau^{-1}(\eta)}|w(\eta)|^{2} d \eta .
$$

Assuming $\tau^{-1}(t) \leq t+h$ (which necessarily implies $\rho(t) \in[0, h], h>0$ ) for all $t \geq-\rho(0)$,

$$
\int_{0}^{t} e^{\lambda s}|w(s-\rho(s))|^{2} d s \leq \frac{e^{\lambda h}}{1-\rho_{*}} \int_{-\rho(0)}^{t} e^{\lambda \eta}|w(\eta)|^{2} d \eta .
$$

Therefore,

$$
\begin{aligned}
e^{\lambda t}|w(t)|^{2} \leq & |w(0)|^{2}+\int_{0}^{t}\left(\lambda \lambda_{1}^{-1}-2 \nu+\lambda_{1}^{-1} M+\frac{l_{1}}{\nu-\lambda_{1}^{-1} M}|f|+\frac{l_{2}}{\nu^{2}\left(\nu-\lambda_{1}^{-1} M\right)^{3}}|f|^{3}\right) e^{\lambda s}\|w(s)\|^{2} d s \\
& +M \int_{0}^{t} e^{\lambda s}|w(s-\rho(s))|^{2} d s \\
\leq & |w(0)|^{2}+\int_{0}^{t}\left(\lambda \lambda_{1}^{-1}-2 \nu+\lambda_{1}^{-1} M+\frac{l_{1}}{\nu-\lambda_{1}^{-1} M}|f|+\frac{l_{2}}{\nu^{2}\left(\nu-\lambda_{1}^{-1} M\right)^{3}}|f|^{3}\right) e^{\lambda s}\|w(s)\|^{2} d s \\
& +\frac{M e^{\lambda h}}{1-\rho_{*}} \int_{0}^{t} e^{\lambda s}|w(s)|^{2} d s+\frac{M e^{\lambda h}}{1-\rho_{*}} \int_{-\rho(0)}^{0} e^{\lambda \eta}|w(\eta)|^{2} d \eta,
\end{aligned}
$$

neglecting the first integral on the right-hand side, which is negative for $0<\lambda \ll 1$ thanks to (4.2),

$$
\begin{aligned}
|w(t)|^{2} & \leq e^{-\lambda t}\left(|w(0)|^{2}+\frac{M e^{\lambda h}}{1-\rho_{*}} \int_{-\rho(0)}^{0} e^{\lambda \eta}|w(\eta)|^{2} d \eta\right) \\
& \leq C e^{-\lambda t}\left(|w(0)|^{2}+\left\|\phi-u_{\infty}\right\|_{L^{2}((-\rho(0), 0) ; H)}\right),
\end{aligned}
$$

where $C=\max \left\{1, M e^{\lambda h} /\left(1-\rho_{*}\right)\right\}$. However, as mentioned before, this argument requires that $\rho(t) \in[0, h]$ is bounded. In other words, we could not prove, in general, the exponential stability of stationary solution to (2.2) with unbounded variable delay by Theorem 4.4.

\subsection{A Razumikhin technique}

In the previous section we have showed the local stability of stationary solutions when the delay operator $g$ contains unbounded variable delay which is driven by a continuously differentiable function $\rho$. However, it is possible to relax this restriction and prove a result for more general delay forcing terms by using a different method, namely, the Razumikhin method, which is also 
widely used in dealing with the stability properties of delay differential equations. But it is worth mentioning that this approach requires some kind of continuity concerning both the operators in the model and the delay term, and we also need to work with strong solutions instead of weak ones.

Theorem 4.7. Consider $f \in\left(L^{2}(\Omega)\right)^{2}$ and $g: \mathbb{R}_{+} \times B C L_{-\infty}(H) \rightarrow\left(L^{2}(\Omega)\right)^{2}$ satisfying conditions $(g 1)-(g 4)$ (uniformly for any $T>0$ ) and such that for all $\xi \in B C L_{-\infty}(H)$ the mapping $\mathbb{R}_{+} \ni t \mapsto g(t, \xi) \in\left(L^{2}(\Omega)\right)^{2}$ is continuous.

Assume that $u_{\infty} \in D(A)$ is a stationary solution to (2.2) such that

$$
\begin{aligned}
& -\nu\left\langle A\left(\psi(0)-u_{\infty}\right), \psi(0)-u_{\infty}\right\rangle-\left\langle B(\psi(0))-B\left(u_{\infty}\right), \psi(0)-u_{\infty}\right\rangle \\
& +\left(g(t, \psi)-g\left(t, u_{\infty}\right), \psi(0)-u_{\infty}\right)<0, \quad t \geq 0
\end{aligned}
$$

for any $\psi \in B C L_{-\infty}(H)$ with $\psi(0) \in V$ and $\psi \neq u_{\infty}$ such that

$$
\left\|\psi-u_{\infty}\right\|_{B C L_{-\infty}(H)}=\left|\psi(0)-u_{\infty}\right|
$$

Then, for any $\phi \in B C L_{-\infty}(H)$ with $\phi(0) \in V$, the solution $u(\cdot ; \phi)$ to (2.2)-(2.3) satisfies

$$
\left|u(t ; \phi)-u_{\infty}\right| \leq\left\|\phi-u_{\infty}\right\|_{B C L_{-\infty}(H)} \forall t \geq 0 .
$$

Proof. The case $\phi=u_{\infty}$ is trivial. Thus assume that $\phi \neq u_{\infty}$. We argue by contradiction. Suppose there exists an initial datum $\phi \in B C L_{-\infty}(H)$ with $\phi(0) \in V$ and $\phi \neq u_{\infty}$, such that (4.8) is false. Then, there exists $t>0$ with $\left|u(t ; \phi)-u_{\infty}\right|>\left\|\phi-u_{\infty}\right\|_{B C L_{-\infty}(H)}$. Denoting

$$
\sigma=\inf \left\{t>0:\left|u(t, \phi)-u_{\infty}\right|>\left\|\phi-u_{\infty}\right\|_{B C L_{-\infty}(H)}\right\},
$$

we obtain for all $0 \leq s \leq \sigma$ that

$$
\left|u(s ; \phi)-u_{\infty}\right| \leq\left|u(\sigma ; \phi)-u_{\infty}\right|=\left\|\phi-u_{\infty}\right\|_{B C L_{-\infty}(H)},
$$

and there is a sequence $\left\{t_{k}\right\}_{k \geq 1} \subset(\sigma, \infty)$ such that $t_{k} \downarrow \sigma$, as $k \rightarrow \infty$, and

$$
\left|u\left(t_{k} ; \phi\right)-u_{\infty}\right|>\left|u(\sigma ; \phi)-u_{\infty}\right|
$$

On the other hand, by virtue of (4.9) it is easy to deduce that

$$
\sup _{\theta \leq 0}\left|u(\sigma+\theta ; \phi)-u_{\infty}\right|=\left\|u_{\sigma}-u_{\infty}\right\|_{B C L_{-\infty}(H)}=\left|u(\sigma ; \phi)-u_{\infty}\right|
$$

which, on account of assumption (4.6)-(4.7) with $\psi=u_{\sigma}$, immediately implies

$$
\begin{aligned}
& -\nu\left\langle A\left(u(\sigma ; \phi)-u_{\infty}\right), u(\sigma ; \phi)-u_{\infty}\right\rangle-\left\langle B(u(\sigma ; \phi))-B\left(u_{\infty}\right), u(\sigma ; \phi)-u_{\infty}\right\rangle \\
& +\left(g\left(\sigma, u_{\sigma}(\cdot ; \phi)\right)-g\left(t, u_{\infty}\right), u(\sigma ; \phi)-u_{\infty}\right)<0
\end{aligned}
$$

By the continuity of the operators in the problem, there exists $\epsilon>0$ such that for all $t \in[\sigma, \sigma+\epsilon]$

$$
\begin{aligned}
& -\nu\left\langle A\left(u(t ; \phi)-u_{\infty}\right), u(t ; \phi)-u_{\infty}\right\rangle-\left\langle B(u(t ; \phi))-B\left(u_{\infty}\right), u(t ; \phi)-u_{\infty}\right\rangle \\
& +\left(g\left(t, u_{t}(\cdot ; \phi)\right)-g\left(t, u_{\infty}\right), u(t ; \phi)-u_{\infty}\right)<0
\end{aligned}
$$


Denoting $w(t)=u(t ; \phi)-u_{\infty}$

$$
\frac{1}{2} \frac{d}{d t}|w(t)|^{2}=-\nu\langle A w(t), w(t)\rangle-\left\langle B(u(t, \phi))-B\left(u_{\infty}\right), w(t)\right\rangle+\left(g\left(t, u_{t}\right)-g\left(t, u_{\infty}\right), w(t)\right)<0
$$

for all $t \in[\sigma, \sigma+\epsilon]$. Therefore, taking $t_{k(\epsilon)} \in(\sigma, \sigma+\epsilon]$,

$$
\begin{aligned}
\left|w\left(t_{k(\epsilon)} ; \phi\right)\right|^{2}-|w(\sigma ; \phi)|^{2}= & 2 \int_{\sigma}^{t_{k(\epsilon)}}-\nu\langle A w(t), w(t)\rangle-\left\langle B(u(t, \phi))-B\left(u_{\infty}\right), w(t)\right\rangle d t \\
& +2 \int_{\sigma}^{t_{k(\epsilon)}}\left(g\left(t, u_{t}\right)-g\left(t, u_{\infty}\right), w(t)\right) d t<0 .
\end{aligned}
$$

Thus $\left|w\left(t_{k(\epsilon)} ; \phi\right)\right|<|w(\sigma ; \phi)|$, which contradicts (4.10). Hence (4.8) is true.

Remark 4.8. (i) The above result is valid even without uniqueness of stationary solution.

(ii) In the spirit of Example 4.1, this result can be applied when $g(t, \xi):=G(\xi(-\rho(t)))$ for $(t, \xi) \in \mathbb{R}_{+} \times B C L_{-\infty}(H)$, with $\rho \in C\left(\mathbb{R}_{+} ; \mathbb{R}_{+}\right)$.

A sufficient condition which implies (4.6) but easier to check in applications is proved in the next result.

Corollary 4.9. Suppose that $f$ and $g$ satisfy assumptions of Theorem 4.4. If

$$
2 \nu>2 \lambda_{1}^{-1} M+\frac{l_{1}}{\nu-\lambda_{1}^{-1} M}|f|+\frac{l_{2}}{\nu^{2}\left(\nu-\lambda_{1}^{-1} M\right)^{3}}|f|^{3},
$$

where $l_{1}, l_{2}$ are defined in (4.5), then there exists at least one solution $u_{\infty} \in D(A)$ to (4.1). Moreover, for all $\phi \in B C L_{-\infty}(H)$ with $\phi(0) \in V$ and $\phi \neq u_{\infty}$, the strong solution $u(t ; \phi)$ to (2.2)-(2.3), satisfies (4.8).

Proof. Since $\nu>\lambda_{1}^{-1} M$, existence of stationary solution is guaranteed by Theorem $4.2(a)$.

For the second statement we check that condition (4.11) implies the ones of Theorem 4.7. Indeed, suppose that $\phi \in B C L_{-\infty}(H)$, with $\phi(0) \in V$, is close to some stationary solution $u_{\infty}$ (but not equal, otherwise it is trivial) and satisfies

$$
\left\|\phi-u_{\infty}\right\|_{B C L_{-\infty}(H)}^{2}=\left|\phi(0)-u_{\infty}\right|^{2} .
$$

Now we verify that (4.6) holds. Indeed

$$
\begin{aligned}
& -\nu\left\langle A\left(\phi(0)-u_{\infty}\right), \phi(0)-u_{\infty}\right\rangle-\left\langle B(\phi(0))-B\left(u_{\infty}\right), \phi(0)-u_{\infty}\right\rangle+\left(g(t, \phi)-g\left(t, u_{\infty}\right), \phi(0)-u_{\infty}\right) \\
\leq & -\nu\left\|\phi(0)-u_{\infty}\right\|^{2}-b\left(\phi(0)-u_{\infty}, u_{\infty}, \phi(0)-u_{\infty}\right)+M\left\|\phi-u_{\infty}\right\|_{B C L_{-\infty}(H)}\left|\phi(0)-u_{\infty}\right| \\
\leq & -\nu\left\|\phi(0)-u_{\infty}\right\|^{2}+\lambda_{1}^{-1} M\left\|\phi(0)-u_{\infty}\right\|^{2}+\left|b\left(\phi(0)-u_{\infty}, u_{\infty}, \phi(0)-u_{\infty}\right)\right| .
\end{aligned}
$$

By similar computations to those in the proof of Theorem 4.4

$$
\begin{aligned}
& -\nu\left\langle A\left(\phi(0)-u_{\infty}\right), \phi(0)-u_{\infty}\right\rangle-\left\langle B(\phi(0))-B\left(u_{\infty}\right), \phi(0)-u_{\infty}\right\rangle+\left(g(t, \phi)-g\left(t, u_{\infty}\right), \phi(0)-u_{\infty}\right) \\
\leq & \left(-\nu+\lambda_{1}^{-1} M+\frac{l_{1}}{2\left(\nu-\lambda_{1}^{-1} M\right)}|f|+\frac{l_{2}}{2 \nu^{2}\left(\nu-\lambda_{1}^{-1} M\right)^{3}}|f|^{3}\right)\left\|\phi(0)-u_{\infty}\right\|^{2},
\end{aligned}
$$

which is negative by (4.11). Thus, (4.6) holds and therefore (4.8) as well.

Remark 4.10. Observe that the Razumikhin technique only requires continuity on the delay term and the operators of the model. Here (4.11) allows more choices for $\nu$ ensuring stability than the values provided by condition (4.2). In other words, this latter result improves the former one. 


\subsection{Stability and asymptotic stability via the construction of Lyapunov functionals}

In this paragraph we analyze the stability of a very particular stationary solution to (2.2) by constructing Lyapunov functionals. Namely we assume that the stationary solution is the trivial one, of course modifying suitably the assumptions on $f$ and $g$. We start by recalling a result, borrowed from [15], which is the key to prove the result concerning the construction of Lyapunov functionals. To this end, let us introduce an abstract problem. Consider operators $\tilde{A}(t, \cdot): V \rightarrow V^{\prime}$ and $\tilde{f}(t, \cdot): B C L_{-\infty}(H) \rightarrow H$ with $\tilde{A}(t, 0)=0$ and $\tilde{f}(t, 0)=0$. Assume that

$$
\begin{aligned}
& \frac{d u}{d t}=\tilde{A}(t, u)+\tilde{f}\left(t, u_{t}\right) \forall t>0, \\
& u(s)=\phi(s), \quad s \in(-\infty, 0],
\end{aligned}
$$

is a well-posed problem and the solution is continuous in time with values in $H$.

The stability of the trivial solution to (4.12) can be analyzed by constructing Lyapunov functionals (cf. [15, Theorem 1.1]). Moreover, if we improve the decay of the functional, we gain not only stability but asymptotic stability. Namely, we have the following result.

Proposition 4.11. Assume that there exist a functional $U: \mathbb{R}_{+} \times B C L_{-\infty}(H) \rightarrow \mathbb{R}_{+}$and positive numbers $\gamma_{1}, \gamma_{2}$ such that, for any $\phi \in B C L_{-\infty}(H)$, the solution $u(\cdot)=u(\cdot ; \phi)$ to (4.12) satisfies

$$
\begin{aligned}
& U\left(t, u_{t}\right) \geq \gamma_{1}|u(t)|^{2} \forall t \geq 0, \\
& U\left(0, u_{0}\right) \leq \gamma_{2}\|\phi\|_{B C L_{-\infty}(H)}^{2}
\end{aligned}
$$

Then:

(a) If $\frac{d}{d t} U\left(t, u_{t}\right) \leq 0$ for $t \geq 0$, the trivial solution to (4.12) is stable.

(b) If there exists a positive number $\gamma_{3}$ such that $\frac{d}{d t} U\left(t, u_{t}\right) \leq-\gamma_{3}|u(t)|^{2}$ for $t \geq 0$, the trivial solution to (4.12) is asymptotically stable.

Proof. For the first statement, observe that the non-increasing character of $U\left(t, u_{t}\right)$ and the first two conditions give

$$
\gamma_{1}|u(t)|^{2} \leq U\left(t, u_{t}\right) \leq U\left(0, u_{0}\right) \leq \gamma_{2}\|\phi\|_{B C L_{-\infty}(H)}^{2} .
$$

This implies the desired stability,

$$
|u(t)|^{2} \leq \frac{\gamma_{2}}{\gamma_{1}}\|\phi\|_{B C L_{-\infty}(H)}^{2} \forall t \geq 0 .
$$

For the second statement, if $\frac{d}{d t} U\left(t, u_{t}\right) \leq-\gamma_{3}|u(t)|^{2}$, we deduce that

$$
\int_{0}^{\infty}|u(s)|^{2} d s \leq \frac{\gamma_{2}}{\gamma_{3}}\|\phi\|_{B C L_{-\infty}(H)}^{2}
$$

The continuity in time of the solution, with values in $H$, combined with the stability inequality proved previously, implies the asymptotic stability of the trivial solution, i.e. $\lim _{t \rightarrow \infty} u(t)=0$.

We will apply the above result to the following equation, which is a particular case of (4.12).

$$
\frac{d u}{d t}=\tilde{A}(t, u)+F(u(t-\rho(t)))
$$

where $\tilde{A}(t, \cdot): V \rightarrow V^{\prime}$ and $F: H \rightarrow H$ are appropriate operators. The following result is a slight variation of [15, Theorem 2.1]. 
Theorem 4.12. Assume that operators in (4.13) satisfy

$$
\begin{aligned}
& \langle\tilde{A}(t, u), u\rangle \leq-\gamma\|u\|^{2}, \quad \gamma>0, \\
& F: H \rightarrow H, \quad|F(u)| \leq \alpha|u|, u \in V, \\
& \rho \in C^{1}\left(\mathbb{R}_{+} ; \mathbb{R}_{+}\right), \quad \rho^{\prime}(t) \leq \rho_{*}<1 .
\end{aligned}
$$

Then the trivial solution to (4.13) is stable (resp. asymptotically stable) provided that

$$
\gamma \geq \frac{\alpha}{\lambda_{1} \sqrt{1-\rho_{*}}}\left(\text { resp. } \gamma>\frac{\alpha}{\lambda_{1} \sqrt{1-\rho_{*}}}\right) .
$$

Proof. We construct $U: \mathbb{R}_{+} \times B C L_{-\infty}(H) \rightarrow \mathbb{R}_{+}$for (4.13) in the form

$$
U(t, \phi)=|\phi(0)|^{2}+\frac{c}{1-\rho_{*}} \int_{-\rho(t)}^{0}|\phi(s)|^{2} d s,
$$

where $c>0$ is a constant to be determined later on, such that $U$ is a Lyapunov functional. Denoting by $U(t)=U\left(t, u_{t}(\cdot ; \phi)\right)$, where $u_{t}(\cdot ; \phi)$ is the solution to (4.13) with initial value $\phi$, we have $U(t)=|u(t)|^{2}+\frac{c}{1-\rho_{*}} \int_{t-\rho(t)}^{t}|u(s)|^{2} d s$. Consequently,

$$
\begin{aligned}
\frac{d}{d t} U(t) & =2\langle\tilde{A}(t, u(t))+F(u(t-\rho(t))), u(t)\rangle+\frac{c}{1-\rho_{*}}|u(t)|^{2}-\frac{c\left(1-\rho^{\prime}(t)\right)}{\left(1-\rho_{*}\right)}|u(t-\rho(t))|^{2} \\
& \leq-2 \gamma\|u(t)\|^{2}+2 \alpha\left|u ( t - \rho ( t ) ) \left\|\left.u(t)\left|+\frac{c}{\lambda_{1}\left(1-\rho_{*}\right)}\|u(t)\|^{2}-c\right| u(t-\rho(t))\right|^{2}\right.\right. \\
& \leq\left(-2 \gamma+\lambda_{1}^{-1}\left(\frac{c}{1-\rho_{*}}+\frac{\alpha^{2}}{c}\right)\right)\|u(t)\|^{2},
\end{aligned}
$$

where the Poincaré and Young inequalities have been used. Minimizing the coefficient in brackets in the right-hand side, which is attained for $c=\alpha \sqrt{1-\rho_{*}}$, we conclude that

$$
\frac{d}{d t} U(t) \leq 2\left(-\gamma+\frac{\alpha}{\lambda_{1} \sqrt{1-\rho_{*}}}\right)\|u(t)\|^{2} .
$$

Then, the coefficient in brackets in the right-hand side above is less or equal or strictly less than zero depending on the conditions in (4.14). This, jointly with the Poincaré inequality, yields the good control of $\frac{d}{d t} U(t)$ in order to apply Proposition 4.11. Therefore, both stability and asymptotic stability statements follow respectively.

Now going back to problem (2.2), if we assume that $f \equiv 0$ and $g\left(t, u_{t}\right)=G(u(t-\rho(t)))$ with $G: H \rightarrow\left(L^{2}(\Omega)\right)^{2}$ a Lipschitz continuous function with Lipschitz constant $M>0$ and $G(0)=0$, we deduce from above the following result.

Corollary 4.13. Consider the Navier-Stokes system

$$
\frac{d u}{d t}+\nu A u+B(u)=P G(u(t-\rho(t))) \forall t \geq 0,
$$

where $G: H \rightarrow\left(L^{2}(\Omega)\right)^{2}$ fulfills the above conditions and $\nu>\lambda_{1}^{-1} M$. Then, $u \equiv 0$ is the unique stationary solution. Moreover, it is stable (resp. asymptotically stable) provided that $\nu \geq M /\left(\lambda_{1} \sqrt{1-\rho_{*}}\right)\left(\right.$ resp. $\left.\nu>M /\left(\lambda_{1} \sqrt{1-\rho_{*}}\right)\right)$. 
Proof. The fact that the origin is a solution is trivial by the assumption on $G$. That it is the unique stationary solution follows from Theorem $4.2(\mathrm{c})$. Finally, both stability and asymptotic stability properties of the origin are due to Theorem 4.12. Indeed (4.15) can be set in (4.13) by denoting $\tilde{A}(t, u)=-\nu A u-B(u)$ and $F(u(t-\rho(t)))=P G(u(t-\rho(t)))$ taking $\gamma=\nu$ and $\alpha=M$.

Remark 4.14. Taking $f \equiv 0$ in Theorem 4.4, the trivial solution to (2.2) is stable if $\nu>$ $\frac{\left(2-\rho_{*}\right) \lambda_{1}^{-1} M}{2\left(1-\rho_{*}\right)}$. Since $\frac{\left(2-\rho_{*}\right) \lambda_{1}^{-1} M}{2\left(1-\rho_{*}\right)}>\frac{M}{\lambda_{1} \sqrt{1-\rho_{*}}}$ for $\rho_{*} \in(0,1)$, Corollary 4.13 improves, for this case, the condition established in Theorem 4.4.

\subsection{Polynomial stability: a special unbounded variable delay case}

As mentioned in the introduction, the main goal of this paper is to analyze the stability of stationary solutions to (2.2) in the unbounded variable delay case. Three different methods have been used to study the stability of stationary solution in previous sections. However, instead of exponential stability, only local and asymptotic stability of the stationary solution to (2.2) are obtained. In fact, even for simple ordinary differential equations with unbounded variable delay, for instance, the pantograph equation, in which the delay term is given by $\rho(t)=(1-\lambda) t$ with $0<\lambda<1$, the exponential stability of stationary solution cannot be reached. But, fortunately, in this simple case the polynomial stability of stationary solution can be proved, see $[27,26,1]$ for details. Enlightened by [1], we show that it is still possible to prove the polynomial stability of stationary solution to Navier-Stokes equations with proportional delay, which is a particular case of unbounded variable delay. To this end, we first review the following pantograph equation and some technical lemmas that are used in this framework.

An example of the pantograph equation reads

$$
x^{\prime}(t)=a x(t)+b x(\lambda t) \forall t \geq 0, x(0)=x_{0}, \lambda \in(0,1),
$$

which has been studied in $[27,26,1]$ amongst many others.

The following lemmas will be useful.

Lemma 4.15. (Cf. [1, Lemma 3.4]) Let $a \in \mathbb{R}, b>0$ and $\lambda \in(0,1)$. Assume $x$ is the solution to (4.16) with $x(0)>0$. Suppose $p \in C\left(\mathbb{R}_{+}, \mathbb{R}_{+}\right)$satisfies

$$
D^{+} p(t) \leq a p(t)+b p(\lambda t), \quad t \geq 0,
$$

with $0<p(0)<x(0)$ and where $D^{+}$denotes the Dini derivative. Then $p(t) \leq x(t)$ for all $t \geq 0$.

Lemma 4.16. (Cf. [1, Lemma 3.5]) Let $x$ be a solution to (4.16). If $a<0, b \in \mathbb{R}$, then there exists $C=C(a, b, \lambda)>0$ such that

$$
\limsup _{t \rightarrow \infty} \frac{|x(t)|}{t^{\mu}}=C|x(0)|,
$$

where $\mu \in \mathbb{R}$ obeys

$$
0=a+|b| \lambda^{\mu} .
$$

Then, for some (possibly new) $C=C(a, b, \lambda)>0$, we have

$$
|x(t)| \leq C|x(0)|(1+t)^{\mu}, \quad t \geq 0 .
$$


Observe that if $\mu<0$, then (4.17) implies that zero is the only stationary solution to (4.16) and its polynomial stability. Moreover, the equality in the superior limit means that for the delayed ODE (4.16) the polynomial stability is the optimal result one can obtain. Next we use this idea to prove the polynomial stability of stationary solution to (2.2).

Theorem 4.17. Consider (2.2) with $f \equiv 0, g\left(t, u_{t}\right):=L_{g} u(\lambda t)$ with $0<\lambda<1, L_{g} \in \mathbb{R}$ and $\nu>\lambda_{1}^{-1}\left|L_{g}\right|$. Then the origin is the unique stationary solution and any solution $u$ converges to zero polynomially, namely, there exist $C=C\left(\nu, L_{g}, \lambda\right)>0$ and $\mu<0$ such that

$$
|u(t)|^{2}<C|u(0)|^{2}(1+t)^{\mu} \forall t \geq 0,
$$

where $\mu$ satisfies $\left|L_{g}\right|-2 \nu \lambda_{1}+\left|L_{g}\right| \lambda^{\mu}=0$.

Proof. The uniqueness of the origin as stationary solution follows from Theorem 4.2 (c). Taking the inner product of $(2.2)$ with $u$ in $H$, we obtain

$$
\frac{d}{d t}|u(t)|^{2}+2 \nu\|u(t)\|^{2}=2 L_{g}(u(\lambda t), u(t))
$$

By the Poincaré and Young inequalities we have that

$$
\frac{d}{d t}|u(t)|^{2}+2 \lambda_{1} \nu|u(t)|^{2} \leq\left|L_{g}\right||u(t)|^{2}+\left|L_{g}\right||u(\lambda t)|^{2} .
$$

Denoting by $w(t)=|u(t)|^{2}$,

$$
w^{\prime}(t) \leq\left(-2 \lambda_{1} \nu+\left|L_{g}\right|\right) w(t)+\left|L_{g}\right| w(\lambda t) .
$$

By Lemmas 4.15 and 4.16 , there exist $C=C\left(\nu, L_{g}, \lambda\right)>0$ and $\mu \in \mathbb{R}$ such that

$$
w(t) \leq C w(0)(1+t)^{\mu}
$$

Since $-2 \lambda_{1} \nu+2\left|L_{g}\right|<0$, then $\mu<0$, and the polynomial decay of solutions follows.

Remark 4.18. (i) From Theorem 4.17 we find that, as long as $\nu>\lambda_{1}^{-1}\left|L_{g}\right|$, any solution to (2.2) converges polynomially to zero. In this case, this result improves all the stability results established previously.

(ii) In fact, our result can be extended to a more general case, namely, if the delay term is defined as $g(t, \phi)=G(\phi(-(1-\lambda) t))$, which is also Lipschitz.

(iii) As pointed out in [1], the convergence to equilibria needs not be at an exponential rate for equations with unbounded delay. Namely, in the pantograph equation (4.16) (unbounded variable delay), polynomial stability is optimal (cf. [26, Theorem 3] for more details). We have used the comparison Lemma 4.15 to obtain our polynomial stability result, but the question of obtaining sufficient conditions for the exponential stability of solutions for PDE with unbounded variable delay is still an open problem. This will be investigated in future.

\section{Acknowledgments}

The authors wish to thank the anonymous referee for their careful reading and suggestions, which led to improvements on the presentation of a previous version of the manuscript.

Partially supported by the projects MTM2015-63723-P (MINECO/ FEDER, EU) and P12FQM-1492 (Junta de Andalucía), and by NSF of China (Nos. 11671142, 11371087 and 11571125), Science and Technology Commission of Shanghai Municipality (No. 13dz2260400) and Shanghai Leading Academic Discipline Project (No. B407), respectively. 


\section{References}

[1] J. A. D. Appleby and E. Buckwar, Sufficient conditions for polynomial asymptotic behaviour of the stochastic pantograph equation, Electron. J. Qual. Theory Differ. Equ., Proc. 10'th Coll. Qualitative Theory of Diff. Equ. 2016 (2016), 1-32.

[2] A. V. Babin, Attractors of Navier-Stokes equations, In Handbook of mathematical fluid dynamics, Vol. II, 169-222, North-Holland, Amsterdam, 2003.

[3] A. Babin, A. Mahalov and B. Nicolaenko, Global regularity of 3D rotating Navier-Stokes equations for resonant domains, Indiana Univ. Math. J. 48 (1999), 1133-1176.

[4] A. Babin, A. Mahalov and B. Nicolaenko, Global regularity of 3D rotating Navier-Stokes equations for resonant domains, Appl. Math. Lett. 13 (2000), 51-57.

[5] T. Caraballo and X. Y. Han, Stability of stationary solutions to 2D-Navier-Stokes models with delays, Dyn. Partial Differ. Equ. 11 (2014), 345-359.

[6] T. Caraballo and X. Y. Han, A survey on Navier-Stokes models with delays: existence, uniqueness and asymptotic behavior of solutions, Discrete Contin. Dyn. Syst. Ser. S 8 (2015), $1079-1101$.

[7] T. Caraballo, A. M. Márquez-Durán and J. Real, Pullback and forward attractors for a 3D LANS- $\alpha$ model with delay, Discrete Contin. Dyn. Syst. 15 (2006), 559-578.

[8] T. Caraballo, A. M. Márquez-Durán and J. Real, Asymptotic behaviour of the threedimensional $\alpha$-Navier-Stokes model with delays, J. Math. Anal. Appl. 340 (2008), 410-423.

[9] T. Caraballo, A. M. Márquez-Durán and J. Real, Asymptotic behaviour of the threedimensional $\alpha$-Navier-Stokes model with locally Lipschitz delay forcing terms, Nonlinear Anal. 71 (2009), e271-e282.

[10] T. Caraballo, A. M. Márquez-Durán and J. Real, Three-dimensional system of globally modified Navier-Stokes equations with delay, Internat. J. Bifur. Chaos Appl. Sci. Engrg. 20 (2010), 2869-2883.

[11] T. Caraballo and J. Real, Navier-Stokes equations with delays, R. Soc. Lond. Proc. Ser. A Math. Phys. Eng. Sci. 457 (2001), 2441-2453.

[12] T. Caraballo and J. Real, Asymptotic behaviour of two-dimensional Navier-Stokes equations with delays, R. Soc. Lond. Proc. Ser. A Math. Phys. Eng. Sci. 459 (2003), 3181-3194.

[13] T. Caraballo and J. Real, Attractors for 2D-Navier-Stokes models with delays, J. Differential Equations 205 (2004), 271-297.

[14] T. Caraballo and J. Real, Asymptotic behaviour and attractors of 2D-Navier-Stokes models with delays, In EQUADIFF 2003, 827-832, World Sci. Publ., Hackensack, NJ, 2005.

[15] T. Caraballo, J. Real and L. Shaikhet, Method of Lyapunov functionals construction in stability of delay evolution equations, J. Math. Anal. Appl. 334 (2007), 1130-1145.

[16] P. Constantin and C. Foias, Navier-Stokes equations, University of Chicago Press, Chicago, IL, 1988. 
[17] C. Foias, O. Manley, R. Rosa and R. Temam, Navier-Stokes equations and turbulence, Cambridge University Press, Cambridge, 2001.

[18] J. García-Luengo, P. Marín-Rubio and G. Planas, Attractors for a double time-delayed 2DNavier-Stokes model, Discrete Contin. Dyn. Syst. 34 (2014), 4085-4105.

[19] J. García-Luengo, P. Marín-Rubio and J. Real, Pullback attractors for 2D Navier-Stokes equations with delays and their regularity, Adv. Nonlinear Stud. 13 (2013), 331-357.

[20] J. García-Luengo, P. Marín-Rubio and J. Real, Regularity of pullback attractors and attraction in $H^{1}$ in arbitrarily large finite intervals for 2D Navier-Stokes equations with infinite delay, Discrete Contin. Dyn. Syst. 34 (2014), 181-201.

[21] J. García-Luengo, P. Marín-Rubio and J. Real, Some new regularity results of pullback attractors for 2D Navier-Stokes equations with delays, Commun. Pure Appl. Anal. 14 (2015), $1603-1621$.

[22] M. J. Garrido-Atienza and P. Marín-Rubio, Navier-Stokes equations with delays on unbounded domains, Nonlinear Anal. 64 (2006), 1100-1118.

[23] J. K. Hale and J. J. Kato, Phase space for retarded equations with infinite delay, Funkcial. Ekvac. 21 (1978), 11-41.

[24] J. K. Hale and S. M. Verduyn Lunel, Introduction to functional-differential equations, Springer-Verlag, New York, 1993.

[25] Y. Hino, S. Murakami and T. Naito, Functional-differential equations with infinite delay, Springer-Verlag, Berlin, 1991.

[26] T. Kato, Asymptotic behavior of solutions of the functional differential equation $y^{\prime}(x)=$ $a y(\lambda x)+b y(x)$, In Delay and functional differential equations and their applications (Proc. Conf., Park City, Utah, 1972), 197-217, Academic Press, New York, 1972.

[27] T. Kato and J. B. McLeod, The functional-differential equation $y^{\prime}(x)=a y(\lambda x)+b y(x)$, Bull. Amer. Math. Soc. 77 (1971), 891-937.

[28] O. A. Ladyzhenskaya, Solution "in the large" to the boundary-value problem for the NavierStokes equations in two space variables, Soviet Physics. Dokl. 123 (1958), 1128-1131.

[29] P. Marín-Rubio, A. M. Márquez-Durán and J. Real, Three dimensional system of globally modified Navier-Stokes equations with infinite delays, Discrete Contin. Dyn. Syst. Ser. B 14 (2010), 655-673.

[30] P. Marín-Rubio, A. M. Márquez-Durán and J. Real, On the convergence of solutions of globally modified Navier-Stokes equations with delays to solutions of Navier-Stokes equations with delays, Adv. Nonlinear Stud. 11 (2011), 917-927.

[31] P. Marín-Rubio, A. M. Márquez-Durán and J. Real, Pullback attractors for globally modified Navier-Stokes equations with infinite delays, Discrete Contin. Dyn. Syst. 31 (2011), 779-796.

[32] P. Marín-Rubio, A. M. Márquez-Durán and J. Real, Asymptotic behavior of solutions for a three dimensional system of globally modified Navier-Stokes equations with a locally Lipschitz delay term, Nonlinear Anal. 79 (2013), 68-79. 
[33] P. Marín-Rubio and J. Real, Attractors for 2D-Navier-Stokes equations with delays on some unbounded domains, Nonlinear Anal. 67 (2007), 2784-2799.

[34] P. Marín-Rubio and J. Real, Pullback attractors for 2D-Navier-Stokes equations with delays in continuous and sub-linear operators, Discrete Contin. Dyn. Syst. 26 (2010), 989-1006.

[35] P. Marín-Rubio, J. Real and J. Valero, Pullback attractors for a two-dimensional NavierStokes model in an infinite delay case, Nonlinear Anal. 74 (2011), 2012-2030.

[36] S. Murakami, Stability in functional-differential equations with infinite delay, Tohoku Math. J. (2), 37 (1985), 561-570.

[37] T. Naito, On linear autonomous retarded equations with an abstract phase space for infinite delay, J. Differential Equations 33 (1979), 74-91.

[38] R. Temam, Navier-Stokes equations. Theory and numerical analysis, AMS Chelsea Publishing, Providence, RI, 2001.

[39] M. J. Wei and T. Zhang, Exponential stability for stochastic 2D-Navier-Stokes equations with time delay, Appl. Math. J. Chinese Univ. Ser. A 24 (2009), 493-500. 